\title{
A IMPORTÂNCIA DAS FLORESTAS PARA AS CRIANÇAS DA REGIÃO DO ALTO URUGUAI, RIO GRANDE DO SUL
}

The importance of forests for children in the region of the Alto Uruguai, Rio Grande do Sul

\author{
Dienifer Calgarotto $^{1 ;}$ Sônia Beatris Balvedi Zakrzevski²
}

\footnotetext{
${ }^{1}$ Graduada em Ciências Biológicas. URI Erechim. E-mail: dieni_calgarotto@hotmail.com.br

${ }^{2}$ Graduada em Ciências. Mestre em Educação e Doutora em Ecologia. Professora do Departamento de Ciências Biológicas da URI Erechim
}

Data do recebimento: $14 / 10 / 2020$ - Data do aceite: 03/11/2020

\begin{abstract}
RESUMO: As florestas, ricas em biodiversidade, contribuem com vários serviços ecossistêmicos cruciais e são de grande importância para a sociedade humana. Este estudo tem por objetivo identificar e analisar as percepções de crianças, residentes na Região do Alto Uruguai do RS, sobre o conceito e sobre a importância das florestas, verificando os fatores que interferem sobre as suas percepções. Participaram do estudo 200 estudantes de $5^{\circ}$ ano do Ensino Fundamental, sendo 100 estudantes residentes em municípios predominantemente urbanos (MPU) e 100 estudantes em municípios predominantemente rurais (MPR). A coleta dos dados foi realizada por meio de um questionário, constituído por questões abertas e fechadas, que possibilitaram a caracterização socioeconômica e cultural dos participantes, bem como a identificação da percepção sobre as florestas e sobre os serviços ecossistêmicos por elas prestados pelas florestas. Após, os dados de cada pergunta foram submetidos a um processo de análise de conteúdo e à análise estatística. As crianças conceituam a floresta como um espaço ocupado pelas plantas e animais, sem a presença humana. Reconhecem que as florestas são responsáveis por inúmeras funções ambientais, com destaque para as funções de regulação e provisão. Cabe à educação formal contribuir na ampliação dos saberes e valores associados às florestas.
\end{abstract}

Palavras-chave: Percepção Ambiental. Educação. Serviços Ecossistêmicos. 


\begin{abstract}
Forests, rich in biodiversity, contribute to several crucial ecosystem services and are of great importance to human society. The objective of this study is to identify and analyze the perceptions of children, residents of the Alto Uruguai Region, in the state of Rio Grande do Sul, on the concept and importance of forests, verifying the factors that interfere with their perceptions. 200 students from the 5th grade of Elementary School participated in this study, 100 students living in Predominantly Urban Region, and 100 students living in Predominantly Rural Region. Data collection was carried out through a questionnaire, consisting of open and closed questions, which enabled the socioeconomic and cultural characterization of the participants, as well as the identification of the perception about forests and the ecosystem services provided by them. After that, the data of each question was submitted to a process of content and statistical analysis. Children conceptualize the forest as a space occupied by plants and animals, without human presence. They recognize that forests are responsible for numerous environmental functions, with emphasis on regulation and provision functions. It is the responsibility of formal education to contribute to the expansion of knowledge and values associated with forests.
\end{abstract}

Keywords: Environmental Perception. Education. Ecosystem Services.

\section{Introdução}

A preocupação com a conservação das florestas é antiga. A Organização das Nações Unidas para a Alimentação e a Agricultura FAO, monitora as florestas do mundo desde 1946, por meio de avaliações realizadas pelo Global Forest Resources Assessment - FRA, descrevendo o estado das florestas do mundo e como elas estão alterando quantitativamente ao longo do tempo (FAO, 2018). Mas foi com a aprovação da Convenção da Diversidade Biológica - CDB, em 1992, que o tema ganha visibilidade em nível global. O Plano Estratégico para a Biodiversidade 2011-2020, conhecido como Metas de Aichi, estabeleceu como um dos seus objetivos reduzir em, pelo menos, $50 \%$ a taxa de perda de todos os habitats naturais, incluindo as florestas (UNEP, 2010). Em 2017, foi lançado o Plano Estra- tégico das Nações Unidas para as Florestas 2017-2030, com a intenção de "aumentar as áreas de florestas com ações de proteção, restauração, reflorestamento e incentivo à gestão sustentável das áreas de florestas, bem como melhorar as condições econômicas, sociais e ambientais das florestas, inclusive melhorando os meios de subsistência das populações delas dependentes" (UN, 2017).

As florestas, ricas em biodiversidade, cobrem $31 \%$ da área terrestre do Planeta (FAO, 2020). Elas compreendem alguns dos habitats mais ricos em espécies do mundo (LEADLEY et al., 2010; HOFFMANN et al., 2010; MEIJAARD et al., 2013), abrigando cerca de $90 \%$ de todas as espécies terrestres de seres vivos do Planeta (UN, 2017). Aproximadamente 1,6 bilhão de pessoas $(25 \%$ da população global) depende diretamente das florestas para viver. E desempenham um papel fundamental no combate à pobreza rural, garantem a segurança alimentar e propor- 
cionam meios de subsistência às populações humanas (UN, 2015; FAO, 2020).

As florestas também contribuem com inúmeros serviços ecossistêmicos (MILLENNIUM ECOSYSTEM ASSESSMENT, 2005; HANSEN, STEHMAN; POTAPOV, 2010; GAMFELDT, 2013). Entre os serviços prestados pelas florestas, merecem destaque a formação do solo e ciclagem de nutrientes, equilíbrio hídrico, regulação de microclimas, produção de madeira, alimentos, fibras, fármacos, entre outros (HANSEN, STEHMAN e POTAPOV, 2010; GAMFELD et. al., 2013; MEIJAARD et al., 2013; LIANG et al., 2016). Elas também contribuem na proteção de recursos hídricos, na redução de eventos climáticos extremos como secas, enchentes, deslizamentos de terra (NEDEL, SAUSEN, SAITO, 2012; SINARE et. al., 2016). Segundo a ONU Meio Ambiente (2019) "parar o desflorestamento e restaurar as florestas danificadas, portanto, poderia fornecer até $30 \%$ da solução climática".

As florestas brasileiras compreendem a maior biodiversidade de espécies do Planeta (ALMEIDA, 2016). O País possui, aproximadamente, $58 \%$ do seu território coberto por florestas nativas e plantadas, o que representa a segunda maior área de florestas do mundo, atrás apenas da Rússia. São estimados 497 milhões de hectares de florestas nativas (FAO, 2020) e 10 milhões de hectares de florestas plantadas (IBGE, 2017).

Em nível global, as florestas tropicais estão desaparecendo, como resultado de muitas pressões. E, a perda de biodiversidade florestal altera os ecossistemas, comprometendo sua capacidade de fornecer bens e serviços ambientais, impactando também o bem-estar-humano (SECRETARIADO DA CONVENÇÃO SOBRE DIVERSIDADE BIOLÓGICA, 2014; PIASENTIN; GOIS, 2016). No Brasil, as principais causas das perdas das florestas devem-se à expansão da agricultura e da pecuária; à exploração madeireira; à introdução de espécies exóticas, sem considerar o planejamento ambiental; à urbanização, ocupando principalmente regiões litorâneas; às atividades de mineração; aos empreendimentos hidrelétricos; à captura e ao tráfico de animais silvestres e à caça indiscriminada (BRASIL, 2016; JACOBI, 2019).

A conservação das florestas depende de políticas públicas, mas principalmente da ação direta dos seres humanos nos ecossistemas a partir do entendimento, destes, quanto à sua importância (ZAKRZEVSKI, VARGAS, DECIAN, 2020). Inúmeros estudos foram desenvolvidos com o intuito de compreender as percepções de diferentes grupos sociais sobre as florestas. Alguns destes estudos são voltados à percepção de comunidades sobre uma gama de serviços ambientais (OUKO et al., 2018; FRICK, BAUER, LINDERN, HUNZIKER, 2018; ZAKRZEVSKI, VARGAS, DECIAN, 2020); outros tratam sobre uma categoria específica, como por exemplo, sobre os serviços culturais (PLIENINGER, DIJKS, OTEROS-ROZAS e BIELING, 2013; ALLENDORF, BRANDT, YANG, 2014). E alguns verificaram a influência dos fatores sociais, econômicos e culturais, na forma como o ser humano percebe o ambiente da floresta (ALLENDORF, BRANDT, YANG, 2014; QUINTAS-SORIANO et al., 2018; ZAKRZEVSKI, VARGAS, DECIAN, 2020) e sobre a influência do local de residência sobre as percepções (MAREN, BHATTARAI, CHAUDHARY 2014; LÓPEZ-SANTIAGO et al., 2014).

$\mathrm{Na}$ última década, pesquisadores brasileiros têm se preocupado com o estudo das percepções de estudantes (crianças e adolescentes) sobre os biomas situados no território nacional (SCHWARZ et al., 2007; PROFICE et al., 2013; SOUZA; SILVA, 2017; VIEIRA et al., 2015; ZAKRZEVSKI, PARIS, DECIAN, 2020; ZANINI et al., 2020). Porém, não há pesquisas sobre a percepção 
de crianças sobre as florestas. Segundo CVETKOVIĆ et al. (2018), o conhecimento da compreensão das crianças sobre os fenômenos florestais é importante para diversos setores como educadores, formuladores de políticas, ambientalistas, entre outros.

Este estudo tem por objetivo identificar e analisar as percepções de crianças, residentes na Região do Alto Uruguai do RS, sobre o conceito e sobre a importância das florestas, verificando os fatores que interferem sobre as suas percepções.

\section{Material e Métodos}

$\mathrm{O}$ estudo foi realizado na região do Alto Uruguai, situada no Norte do Rio Grande do Sul, Sul do Brasil (Figura 1). A região é composta por 32 municípios e corresponde a, aproximadamente, $2,03 \%$ da área do Estado do Rio Grande do Sul, com $5745 \mathrm{~km}^{2}$ (IBGE, 2017). Ela pertence ao Bioma Mata Atlântica, possuindo um complexo mosaico de diferentes formações vegetais.

A vegetação original apresenta dois traços distintos: ao Norte, Floresta Ombrófila Mista, ou também chamada de Floresta de Araucária; ao Sul, Floresta Estacional Decidual e também vegetação campestre. E, conforme Piran (2015), atualmente encontra-se bastante devastada, principalmente no Sul, em decorrência da ocupação humana e da implantação da agricultura moderna, enquanto ao Norte, o êxodo rural dos pequenos agricultores, a acidentação do relevo e as restrições legais à utilização dos solos vêm provocando um processo de recuperação da cobertura vegetal.

Dentre os 32 municípios da região, foram sorteados cinco municípios para participação na pesquisa: i) um município predominantemente urbano (MPU), ou seja, que possui mais de 50 mil habitantes, em área de ocupação densa; ii) quatro municípios predominan- temente rurais (MPR) que possuem entre 3 mil e 10 mil habitantes em área de ocupação densa, com grau de urbanização inferior a $75 \%$. Do MPU foram sorteadas cinco escolas públicas e uma escola privada e dos MPR foi sorteada uma escola por município.

Figura I - Localização da Região do Alto Uruguai, RS.

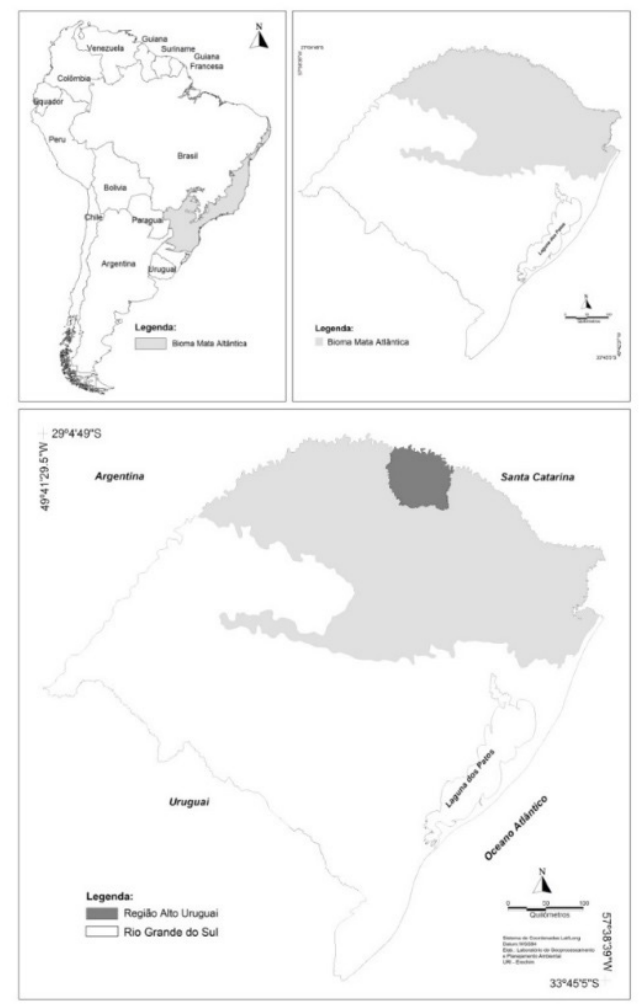

Fonte: Laboratório de Geoprocessamento e Planejamento Ambiental - URI

A coleta dos dados foi realizada por meio de um questionário, aplicado em data, local e horário definidos com as direções das escolas, após a aprovação do projeto pelo Comitê de Ética em Pesquisa, da URI - Erechim (Parecer 3.392.530/2019), e consentimento dos pais e/ou responsáveis pelos participantes, por meio da assinatura de um Termo de Consentimento Livre e Esclarecido e do assentimento das crianças. 
Tabela I - Caracterização das crianças do $5^{\circ}$ ano de Ensino Fundamental, residentes na Região do Alto Uruguai, RS, organizadas segundo categorias de municípios, sexo e local de residência

\begin{tabular}{c|c|c|c|c}
\hline \multirow{2}{*}{ Categorias de municípios } & \multicolumn{2}{|c|}{ Sexo } & \multicolumn{2}{c}{ Local de Residência } \\
\cline { 2 - 5 } & $\begin{array}{c}\text { Masculino } \\
(\mathrm{n}=91)\end{array}$ & $\begin{array}{c}\text { Feminino } \\
(\mathrm{n}=109)\end{array}$ & $\begin{array}{c}\text { Urbano } \\
(\mathrm{n}=151)\end{array}$ & $\begin{array}{c}\text { Rural } \\
(\mathrm{n}=49)\end{array}$ \\
\hline MPR: $>$ 5.000 hab. $(\mathrm{n}=90)$ & 48 & 52 & 56 & 44 \\
\hline MPU: 3.000- 10.000 hab. $(\mathrm{n}=100)$ & 43 & 57 & 95 & 5 \\
\hline
\end{tabular}

O instrumento de pesquisa foi constituído por questões abertas e fechadas, que possibilitaram a caracterização socioeconômica e cultural dos participantes, bem como a identificação da percepção sobre as florestas e sobre os serviços ecossistêmicos por elas prestados pelas florestas. Após, os dados de cada pergunta foram submetidos a um processo de análise de conteúdo (BARDIN, 1977). O conjunto de dados de cada questão foi organizado em planilhas, no Microsoft Excel e submetido a um processo de análise descritiva, com o objetivo de evidenciar as características de distribuição das variáveis. Os dados também foram submetidos ao teste do qui-quadrado $\left(\chi^{2}\right)$ com $p<0,05$, buscando verificar se o local de residência, gênero e tipo de escola que frequentam exerce influência sobre as suas percepções. As análises foram realizadas utilizando-se o software Bioestat 5.0.

\section{Resultados e Discussão}

\section{O conceito de floresta}

As crianças, por meio de pequenos textos expressaram diferentes ideias sobre o que é uma floresta e foram agrupadas em seis categorias. É importante destacar que as crianças expressam mais de uma ideia sobre o tema (Tabela II). Para 57\% das crianças, as florestas são lugares com muitas árvores, espécies de plantas não arbóreas e uma rica diversidade de espécies animais.
Por meio de análise estatística evidenciaram-se diferenças significativas entre as ideias apresentadas pelas crianças que residem no meio urbano e meio rural $(\chi 2$ $=12,44 ; \mathrm{gl}=5 ; \mathrm{p}=0,02)$. Porém, em nenhuma definição as crianças incluíram a presença do ser humano. Isso nos faz pensar que as crianças participantes da pesquisa associam as florestas com um local distante dos lugares que habitam as populações humanas. Ignoram que as florestas são locais onde vivem povos, que delas dependem para a sobrevivência; que algumas comunidades são partes integrantes das florestas e que o seu uso sustentável garante o bem estar humano.

A maioria das crianças (74\%) respondeu que já teve algum contato direto com ambientes de floresta (Tabela III). E, quando comparados os participantes da pesquisa, em relação ao contato com os ambientes florestais, verificou-se diferença significativa, entre as respostas de meninas e meninos $\left(\chi^{2}=7,83\right.$; $\mathrm{gl}=1 ; \mathrm{p}=0,005)$.

Os locais em que as crianças informaram que entraram em contato com os ambientes florestais foram: i) $31,5 \%$, nas propriedades de familiares; ii) $21 \%$, em viagens para outras regiões; iii) 9\%, em Unidades de Conservação situadas no município em que residem; e iv) 4,5\%, na própria escola (Tabela III).

Alguns fatores, provavelmente contribuem para o contato das crianças da Região com ambientes florestais: i) o aumento de áreas de vegetação nativa na Região que passou de 11,66\%, em 1986, para 25,41\%, em 2016, 
Tabela II - Definições de floresta, segundo estudantes do $5^{\circ}$ ano do Ensino Fundamental, residentes na Região do Alto Uruguai, RS, organizados segundo categorias de municípios, sexo e local de residência

\begin{tabular}{ccccccc}
\hline \multirow{2}{*}{ Conceitos de floresta } & \multicolumn{2}{c}{ Município } & \multicolumn{2}{c}{ Sexo } & \multicolumn{2}{c}{ Residência } \\
\cline { 2 - 7 } & MPU & MPR & Fem. & Masc. & Rural & Urbano \\
\cline { 2 - 7 } & $\mathrm{n}=100$ & $\mathrm{n}=100$ & $\mathrm{n}=109$ & $\mathrm{n}=91$ & $\mathrm{n}=49$ & $\mathrm{n}=151$ \\
\hline Lugar com grande biodiversidade (plantas & & & & & & \\
e animais) & 60 & 54 & 67 & 44 & 35 & 76 \\
Lugar com muitas árvores & 21 & 25 & 22 & 23 & 4 & 41 \\
Natureza, com morros e rios & 19 & 15 & 20 & 13 & 9 & 24 \\
Sinônimo de mato & 14 & 5 & 14 & 5 & 3 & 16 \\
É a casa dos animais & 13 & 19 & 14 & 17 & 8 & 23 \\
Um lugar bonito e alegre & 13 & 15 & 14 & 15 & 5 & 24 \\
\hline
\end{tabular}

Tabela III - Ambientes de contato direto com o ambiente de florestas, segundo os estudantes do $5^{\circ}$ ano do Ensino Fundamental, residentes na Região do Alto Uruguai, RS, organizados segundo categorias de municípios, sexo e local de residência

\begin{tabular}{cccccccc}
\hline \multirow{2}{*}{ Contato com o ambiente de floresta } & \multicolumn{2}{c}{ Município } & \multicolumn{2}{c}{ Sexo } & \multicolumn{2}{c}{ Residência } \\
& MPU & MPR & Fem. & Masc. & Rural & Urbano \\
\cline { 2 - 7 } & $\mathrm{n}=100$ & $\mathrm{n}=100$ & $\mathrm{n}=109$ & $\mathrm{n}=91$ & $\mathrm{n}=49$ & $\mathrm{n}=151$ \\
\hline Unidade de conservação situada no município & 4 & 14 & 4 & 13 & 4 & 12 \\
Propriedade da família & 33 & 30 & 35 & 28 & 10 & 53 \\
Área verde situada na Escola & 9 & 0 & 9 & 0 & 8 & 2 \\
Viagem com familiares para outras regiões & 16 & 26 & 16 & 26 & 6 & 37 \\
\hline
\end{tabular}

em função do êxodo rural, abandono de áreas agrícolas, agricultura mecanizada, legislação vigente, entre outras (ROVANI et al., 2020); ii) a existência de Unidades de Conservação na Região, que possibilitam atividades de educação ambiental e de lazer em contato com a natureza; iii) a importância atribuída pelas populações que residem no meio urbano de contato com ambientes naturais rurais.

\section{Fontes de informação sobre as florestas}

As crianças, por meio de 606 citações, listaram 10 fontes de informação sobre as florestas, com uma média de 3,03 fontes por participante. Com destaque é citada a televisão $(76 \%)$, as atividades escolares realizadas durante as aulas (70,5\%) e o diálogo com os familiares (49\%) (Tabela IV).

No cenário brasileiro, a televisão é o meio de comunicação mais utilizado pela população, sendo que apenas 2,9\% dos brasileiros não possuem televisão em seus domicílios (IBGE, 2016) e 77\% assistem diariamente (BRASIL, 2016). E, algumas pesquisas indicam que os meios de comunicação possuem um potencial significativo para influenciar a compreensão e as percepções sobre as questões ambientais (HANSEN, 2010; ANDRADE et al., 2014; FELDMAN, 2016). De acordo com a National Science Board (2014), a TV é também uma fonte líder de notícias científicas em todo o mundo, especialmente fora da Europa e América do Norte. 
Tabela IV - Fontes de informação sobre as florestas, segundo os estudantes do $5^{\circ}$ ano do Ensino Fundamental, residentes na Região do Alto Uruguai, RS, organizados segundo categorias de municípios, sexo e local de residência

\begin{tabular}{ccccccc}
\hline \multirow{2}{*}{$\begin{array}{c}\text { Fontes de informação } \\
\text { sobre florestas }\end{array}$} & \multicolumn{2}{c}{ Município } & \multicolumn{2}{c}{ Sexo } & \multicolumn{2}{c}{ Local de Residência } \\
\cline { 2 - 7 } & MPU & MPR & Fem. & Masc. & Rural & Urbano \\
\cline { 2 - 7 } & $\mathrm{n}=100$ & $\mathrm{n}=100$ & $\mathrm{n}=109$ & $\mathrm{n}=91$ & $\mathrm{n}=49$ & $\mathrm{n}=151$ \\
\hline TV & 61 & 91 & 68 & 82 & 46 & 106 \\
Aulas - escola & 64 & 77 & 71 & 70 & 38 & 103 \\
Conversas com Familiares & 54 & 44 & 57 & 41 & 34 & 64 \\
Rádio & 21 & 20 & 24 & 17 & 10 & 31 \\
Livros diversos & 12 & 34 & 17 & 29 & 9 & 37 \\
Conversa com amigos & 9 & 17 & 12 & 14 & 7 & 19 \\
Internet & 9 & 55 & 12 & 52 & 4 & 60 \\
Visita em Parques & 2 & 0 & 2 & 0 & 2 & 0 \\
Revista & 1 & 17 & 3 & 17 & 1 & 19 \\
Palestras & 0 & 18 & 0 & 18 & 0 & 18 \\
\hline
\end{tabular}

Os dados demonstram que há diferença significativa entre o número de citações entre as crianças que residem nos MPU e MPR $\left(\chi^{2}=59,25 ; \mathrm{gl}=9 ; \mathrm{p}<0.0001\right)-$ as crianças que residem em MPR citam maior número de fontes de informação (média de 3,73 fontes por participante), se comparadas àquelas que moram nos MPU (média de 2,33 fontes). A importância das atividades escolares e da conversa com familiares, na construção de saberes sobre as florestas merece destaque entre as crianças que residem no meio rural - essas fontes foram citadas por $77,55 \%$ e $69,38 \%$ das crianças, respectivamente. Isso acontece, provavelmente, porque as escolas do meio rural ensinam conhecimentos vinculados aos saberes e culturas que as crianças trazem de suas vivências familiares e comunitárias.

Contrariando o que era esperado, a internet foi mais citada pelas crianças dos MPR, demonstrando que crianças que residem nos municípios pesquisados possuem acesso e fazem uso desse recurso de comunicação e informação na busca de conhecimentos sobre o tema. A pesquisa TIC Domicílios (CETIC,
2020) aponta que 53\% da população brasileira, que vive em áreas rurais, declara ser usuária de Internet. Atualmente, como o mundo está cada vez mais tecnológico, as crianças também usam cada vez mais as tecnologias digitais (CABELLO; CLARO; CABELLO-HUTT, 2015). Conforme dos Santos e Paulo (2018), há um crescente uso do acesso à internet pelas crianças que moram no meio rural e uma inversão da ordem geracional, ou seja, são as crianças que ensinam os adultos a usar as novas Tecnologias de Comunicação e Informação (TCI's). Também há diferença na frequência de citação da internet, livros, revistas e palestras, entre os participantes dos dois $\operatorname{sexos}\left(\chi^{2}=426,8 ; \mathrm{gl}=13 ; \mathrm{p}=0,0001\right)$.

Tendo em vista que a televisão é um veículo importante de informação da população brasileira no geral, é necessário que os conteúdos nela veiculados, sejam objeto de estudo no ambiente escolar. Desta forma a escola pode contribuir na reflexão e discussão sobre o conteúdo ambiental divulgado nos meios de comunicação. 


\section{A importância das florestas}

Para 97\% das crianças participantes da pesquisa, as florestas são ecossistemas que apresentam importância e que devem ser conservados (Tabela V).

Por meio de 292 citações, com a média de 1,46 citações por participante, as crianças apresentaram 8 serviços prestados pelas florestas. As ideias das crianças foram associa- dos às Funções Ecossistêmicas, propostas por De Groot (2006) e De Groot et al. (2010): i) Função de provisão ii) Função de regulação; iii) Função de habitat; iv) Função cultural.

O principal serviço prestado pelas florestas, na percepção das crianças, é a purificação do ar (citada por 62\% dos participantes da pesquisa), associando as florestas com a regulação dos gases atmosféricos e com a produção de oxigênio. Também merece

Tabela V - Valor atribuído às florestas pelos estudantes do $5^{\circ}$ ano do Ensino Fundamental, residentes na Região do Alto Uruguai, RS, organizados segundo categorias de municípios, sexo e local de residência

\begin{tabular}{|c|c|c|c|c|c|c|}
\hline \multirow{2}{*}{$\begin{array}{c}\text { Importância das } \\
\text { florestas }\end{array}$} & \multicolumn{2}{|c|}{ Município } & \multicolumn{2}{|c|}{ Sexo } & \multicolumn{2}{|c|}{ Local de Residência } \\
\hline & MPU & MPR & Fem. & Masc. & Rural & Urbano \\
\hline & $\mathrm{n}=100$ & $\mathrm{n}=100$ & $\mathrm{n}=109$ & $\mathrm{n}=91$ & $\mathrm{n}=49$ & $\mathrm{n}=151$ \\
\hline Importantes & 94 & 100 & 103 & 91 & 46 & 148 \\
\hline Não importantes & 1 & 0 & 1 & 0 & 0 & 1 \\
\hline Não sei & 5 & 0 & 5 & 0 & 3 & 2 \\
\hline
\end{tabular}

Tabela VI - Importância atribuída às florestas, categorizadas em funções e serviços ecossistêmicos, pelos estudantes do $5^{\circ}$ ano do Ensino Fundamental, residentes na Região do Alto Uruguai, RS, organizados segundo categorias de municípios, sexo e local de residência

\begin{tabular}{|c|c|c|c|c|c|c|c|}
\hline \multirow{2}{*}{$\begin{array}{c}\text { Funções } \\
\text { ecossistêmicas }\end{array}$} & \multirow{2}{*}{ Serviços citados } & MPU & MPR & Fem. & Masc. & Rural & Urbano \\
\hline & & $\mathrm{n}=100$ & $\mathrm{n}=100$ & $n=109$ & $\mathrm{n}=91$ & $\mathrm{n}=49$ & $\mathrm{n}=151$ \\
\hline \multirow[b]{2}{*}{$\begin{array}{l}\text { Função de } \\
\text { Provisão }\end{array}$} & Fornecem água & 29 & 22 & 29 & 22 & 13 & 38 \\
\hline & $\begin{array}{c}\text { Produção de madeira } \\
\text { e látex }\end{array}$ & 9 & 6 & 9 & 6 & 7 & 8 \\
\hline \multirow{6}{*}{ Função de hábitat } & Fornece alimentos e & & & & & & \\
\hline & frutos & 0 & 17 & 0 & 17 & 0 & 17 \\
\hline & Habitat de animais & 24 & 34 & 26 & 32 & 13 & 45 \\
\hline & Habitat de plantas e & & & & & & \\
\hline & animais & 9 & 5 & 10 & 4 & 6 & 8 \\
\hline & $\begin{array}{c}\text { Purificam o ar e } \\
\text { produzem } \mathrm{O} 2\end{array}$ & 49 & 75 & 57 & 67 & 21 & 103 \\
\hline \multirow[t]{2}{*}{$\begin{array}{l}\text { Função de } \\
\text { regulação }\end{array}$} & $\begin{array}{l}\text { Ajuda a regular o } \\
\text { meio ambiente - "a }\end{array}$ & 11 & 3 & 11 & 3 & 9 & 5 \\
\hline & funcionar direito" & & & & & & \\
\hline \multirow[t]{2}{*}{ Função Cultural } & Lugar de lazer e & & & & & & \\
\hline & contemplação & 2 & 0 & 2 & 0 & 2 & 0 \\
\hline
\end{tabular}


destaque, em função do número de citações, a função de habitat (citada por $36 \%$ das crianças) e a importância das florestas na produção de água (citada por 25,5\%) (Tabela VI). Com frequência esses temas são apresentados pelos livros didáticos de Ciências e também em programas de televisão, que estabelecem relações entre as florestas e o sequestro de Carbono e com a produção de água de qualidade.

Os estudantes que residem em MER e do sexo masculino são aqueles que citaram um maior número de serviços prestados pelas florestas. E são os meninos que residem no meio urbano que citam a importância das florestas na produção de alimentos. Há diferenças entre a porcentagem dos serviços ecossistêmicos citados entre os estudantes que moram em MPU e MPR $\left(\chi^{2}=248,72\right.$; $\mathrm{gl}=7 ; \mathrm{p}=0.0001)$, entre meninos e meninas $\left(\chi^{2}=248.19 ; \mathrm{gl}=7 ; \mathrm{p}=0.0001\right)$ e entre aqueles que residem no meio urbano e rural $\left(\chi^{2}=289.90 ; \mathrm{gl}=7 ; \mathrm{p}=0.0001\right)$.

Sintetizando, as crianças do $5^{\circ}$ ano, participantes da pesquisa, reconhecem a importância das florestas para a vida humana e para a manutenção da diversidade biológica. Para as crianças, a floresta é como um ator, como uma entidade capaz de gerar bens ou benefícios.

Um estudo desenvolvido com adolescentes sobre as percepções a respeito da Reserva Florestal Ducke, situada em Manaus, constatou que a maioria destaca que as florestas possuem valor econômico e medicinal (frutos, madeira, sementes, raízes); ambiental (clima ameno, sombra e ar puro); para atividades como lazer e contemplação (HIGUCHI et al., 2013). A importância das florestas também foi diagnosticada em um estudo desenvolvido por Genç, Demírkaya e Karasakal (2010) com jovens do $7^{\circ}$ ano de Burdur, sul da Turquia. A pesquisa identificou que os participantes compreendem a importância e o valor da floresta na vida hu- mana; percebem o quanto os seres humanos precisam de florestas, e a vida humana não poderia mais existir sem a existência deste tipo de ecossistema.

Já, outra pesquisa desenvolvida com agricultores, residentes no Norte do RS, identificou que esse grupo percebe inúmeros serviços prestados pelas florestas, associados às funções ecossistêmicas de Regulação (regulação climática; filtragem e estocagem de água; quebra-vento; purificação do ar; controle de processos erosivos; polinização; controle biológico; ciclagem de nutrientes; barreira contra poluentes), Provisão (madeira, lenha, fitofármacos, alimentos), Habitat (abrigo para a fauna e flora) e Informação (turismo, lazer) (ZAKRZEVSKI, VARGAS, DECIAN, 2020).

Estudos demonstram que as percepções de crianças e os adolescentes sobre a floresta não fogem a sistemas fundamentados nas experiências vividas individualmente e nos processos socioculturais e ideológicos construídos coletivamente (HIGUCHI; KUHNEN, 2008; KUHNEN e HIGUCHI, 2011). Isso justifica o fato de as crianças citarem um menor número de serviços prestados pelas florestas, se comparados com pessoas de maior idade, que também percebem outros serviços (OUKO et al., 2018; FRICK, BAUER, LINDERN, HUNZIKER, 2018; ZAKRZEVSKI, VARGAS, DECIAN, 2020).

\section{Considerações Finais}

A pesquisa permitiu concluir que as crianças do $5^{\circ}$ ano, que participaram do estudo, conceituam a floresta como um espaço ocupado pelas plantas e animais, sem a presença humana. Para elas, as florestas são responsáveis por funções ambientais, com destaque para a regulação de gases na atmosfera, contribuindo na purificação do 
ar. A pesquisa constatou que um dos fatores que interfere sobre as percepções das crianças é o tipo de município em que residem - crianças que moram em MER apresentam percepções mais amplas sobre a importância das florestas.

É de grande importância que as Escolas contribuam para que, ao longo da educação básica, as crianças e jovens ampliem seus saberes e valores associados às florestas, reconhecendo as conexões entre as florestas, a rica biodiversidade que sustentam e o bem-estar humano. Além de abrigar a imensa biodiversidade, as florestas prestam à humanidade inúmeros serviços ecossistêmicos essenciais: purificação do ar e da água; produção de alimentos, medicamentos, fibras, madeira, entre outros. As florestas contribuem para manter as bacias hidrográficas e influenciam no clima mundial e no regime das chuvas. São importantes sumidouros de carbono, absorvendo milhões de toneladas do gás, anualmente. Porém, vastas áreas de floresta continuam sendo perdidas.

\section{REFERÊNCIAS}

ALMEIDA, D.S. Recuperação ambiental da Mata Atlântica. 3. ed. Ilhéus, BA: Editus, 2016. Disponível em: http://books.scielo.org/id/8xvf4/pdf/almeida-9788574554402-01.pdf. Acesso em: 9 set. 2019.

ALLENDORF, T. D.; BRANDT, J. S.; YANG, J. M. Local perceptions of Tibetan village sacred forests in northwest Yunnan. Biological Conservation, n. 169, p.303-310, 2014,. Disponível em: https://www.sciencedirect.com/science/article/pii/S0006320713004229. Acesso em: 12 set. 2019.

ANDRADE, A.J.P.; DA SILVA, N.M.; DE SOUZA, C.R. As percepções sobre as variações e mudanças climáticas e as estratégias de adaptação dos agricultores familiares do Seridó potiguar. Desenvolvimento e Meio Ambiente, v. 31, p.77-96, 2014.

BRASIL. MINISTÉRIO DO MEIO AMBIENTE. Brasil: $5^{\circ}$ relatório nacional para a Convenção Sobre Diversidade Biológica. Ministério do Meio Ambiente. Secretaria de Biodiversidade e Florestas. Brasília: MMA, 2016.

CABELLO, P; CLARO, M; CABELLO-HUTT, T. Mediação parental no uso de TIC segundo a percepção de crianças e adolescentes brasileiros: reflexões com base na pesquisa TIC kids online Brasil 2014. In: COMITÊ GESTOR DA INTERNET NO BRASIL. Pesquisa sobre o uso da internet por crianças e adolescentes no Brasil: TIC Kids online Brasil 2015. São Paulo: Comitê Gestor da Internet no Brasil, 2016. Disponível em: https://cetic.br/media/docs/publicacoes/2/TIC_ Kids_2015_LIVRO_ELETRONICO.pdf. Acesso em: 4 nov. 2020.

CVETKOVIĆ, V. M. et al. Childrens and youths' knowledge on forest fires: Discrepancies between basic perceptions and reality. Vojno delo, v. 70, n. 1, p. 171-185, 2018, Disponível em: https:// scindeks.ceon.rs/Article.aspx?artid=0042-84261801171C. Acesso em: 12 ago. 2019.

DE GROOT, R. Functions-analyses and valuation as a to assess land use conflicts in planning for sustainable, multi-functional landscapes. Landscapes and Urban Planning, v.75, p.175-186, 2006.

DE GROOT, R.; AIKEMADE, R.; BRAAT, L.; HEIN, L.; WILLEMEN, L. Challenges in integrating the concept of ecosystem services and values in landscape planning, management and decision making. Ecological Complexity, v.7, p.260-272, 2010. 
FAO. The Forest Resources Assessment Programme. FRA 2015 - Terms and Definitions. Rome: Food and Agriculture Organization of the United Nations, 2012. Disponível em: http://www.fao. org/3/ap862e/ap862e00.pdf. Acesso em: 9 set. 2020.

FAO. 1948-2018. Seventy years of FAO's Global Forest Resources Assessment. Historical overview and future prospects. Food and Agriculture Organization of the United Nations. Rome, 2018. Disponível em: http://www.fao.org/3/I8227EN/i8227en.pdf. Acesso em: 12 maio 2020.

FAO. Global Forest Resources Assessment 2020- Key findings. Rome, 2020. Disponível em:https://doi.org/10.4060/ca8753en. Acesso em: 10 set. 2020.

FELDMAN, L. Effects of TV and Cable News Viewing on Climate Change Opinion, Knowledge, and Behavior. Oxford Research Encyclopedia of Climate Science. 2016. Disponível: http:// oxfordre.com/climatescience/view/10.1093/acrefore/9780190228620.001.0001/acrefore9780190228620-e-367.Acesso em: 07 dez. 2018.

FRICK, J., BAUER, N., LINDERN, E.V.; HUNZIKER, M. What forest is in the light of people's perceptions and values: socio-cultural forest monitoring in Switzerland. Geographica Helvetiva, n.73, p.335-345, 2018. Disponível em: https://gh.copernicus.org/articles/73/335/2018/gh-73-3352018.pd. Acesso em: 12 maio 2020.

GAMFELDT, L. et al., Higher levels of multiple ecosystem services are found in forests with more tree species. Nature communications, v. 4, p. 1340, 2013. Disponível em: https://www.nature.com/ articles/ncomms2328. Acesso em:12 set. 2020.

GENÇ, H.; DEMIRKAYA, H.; KARASAKAL,G.. İlköğretim öğrencilerinin ormana ilişkin görüşleri: Nitel bir araştırma. Makufebed, n. 2, p. 150-166, 2010. Disponível em: https://dergipark. org.tr/tr/download/article-file/181656. Acesso em: 2 ago. 2020.

HANSEN, M. C.; STEHMAN, S. V.; POTAPOV, P. V. Quantification of global gross forest cover loss. Proceedings of the National Academy of Sciences, n. 19, v.107, p. 8650-8655, 2010, Disponível em: https://www.pnas.org/content/107/19/8650. Acesso em: 12 ago. 2020.

HIGUCHI, M. I. G.; KUHNEN, A. Percepção e representação ambiental: Métodos e técnicas de investigação para a educação ambiental. In: PINHEIRO, J.Q; GÜNTHER, H. (Org.), Métodos de pesquisa nos estudos pessoa ambiente. São Paulo: Casa do Psicólogo, 2008, p. 181-215.

HOFFMANN, M. et al., The impact of conservation on the status of the world's vertebrates. Science, v. 330, n. 6010, p. 1503-1509, 2010.

IBGE. Produção da Extração Vegetal e da Silvicultura 2017. IBGE, 2017. Disponível em: https:// agenciadenoticias.ibge.gov.br/media/com_mediaibge/arquivos/15f538e $9095614 \mathrm{fc} 3204 \mathrm{f} 828 \mathrm{~b} 22$ fa714.pdf. Acesso em: 01 abr. 2019.

JACOBI, P. R. et al., Editorial N 3/2019 Amazônia Ameaçada. Ambiente \& Sociedade. São Paulo, v. 22, e0005, 2019. Disponível em: http://www.scielo.br/scielo.php?script=sci_arttext\&pid=S1414753X2019000100203\&lng=en\&nrm=iso. Acesso em: 12 oct. 2020

LEADLEY, P., PEREIRA, H.M., ALKEMADE, R., FERNANDEZ-MANJARRÉS, J.F., PROENÇA, V., SCHARLEMANN, J.P.W. \& WALPOLE, M.J. Biodiversity Scenarios: Projections of 21st century change in biodiversity and associated ecosystem services. Secretariat of the Convention on Biological Diversity, Montreal, 2010. Disponível em: ttps:/www.cbd.int/doc/publications/cbd-ts-50en.pdf. Acesso em: 17 ago. 2019.

LIANG, J., CROWTHER, T. W., PICARD, N., WISER, S., ZHOU, M., ALBERTI, G., ET AL. Positive biodiversity-productivity relationship predominant in global forests. Science, 354, aa8957, 2016. Disponível em: https://science.sciencemag.org/content/354/6309/aaf8957. Acesso em: 10 set. 2020 . 
LÓPEZ-SANTIAGO, C., OTEROS-ROZAS, E., MARTÍN-LÓPEZ, B., PLIENINGER, T., GONZÁLEZ MARTÍN, E., \& GONZÁLEZ, J. (2014). Using visual stimuli to explore the social perceptions of ecosystem services in cultural landscapes: the case of transhumance in Mediterranean Spain. Ecology and Society, n. 19, v.2, art. 27, 2014. Disponível em: https://www. ecologyandsociety.org/vol19/iss2/art27/. Acesso em: 3 set. 2020.

MAREN, I. E.; BHATTARAI, K. R.; CHAUDHARY, R. P. Forest ecosystem services and biodiversity in contrasting Himalayan forest management systems. Environmental Conservation, n. 41, p.73-83, 2014.

MEIJAARD, E. et al. People's perceptions about the importance of forests on Borneo. PloS one, v. 8, n. 9, p. e73008, 2013. Disponível em: https://journals.plos.org/plosone/article?id=10.1371/journal. pone.0073008. Acesso em: 3 out. 2019.

\section{MILLENNIUM ECOSYSTEM ASSESSMENT (MEA). Millennium Ecosystem Assessment} synthesis report. Island Press, Washington, D.C., USA, 2005.

NEDEL, A. S.; SAUSEN, T. M.; SAITO, S. M. Zoneamento dos desastres naturais ocorridos no estado do Rio Grande do Sul no período 2003-2009-Parte II: Granizo e Vendaval. Revista Brasileira de Meteorologia, v. 27, n.2, 2012, p. 119-126.

OUKO, C.A.; MULWA, R.; KIBUGI, R.; OWUOR, M.A.; ZAEHRINGER, J.G.; OGUGEN.O. Community Perceptions of Ecosystem Services and the Management of Mt. Marsabit Forest in Northern Kenya. Environments, v. 5, n.11, 2018. Disponível em: https://www.mdpi.com/20763298/5/11/121/htm. Acesso em: 9 set. 2020.

PIASENTIN, F. B.; GÓIS, S. L. Conservação de remanescentes florestais no Brasil: considerações sobre os principais instrumentos de gestão ambiental. Desenvolvimento e Meio Ambiente, v. 36, p. 115-134, 2016.

PLIENINGER, T.; DIJKS, S.; OTEROS-ROZAS, E.; BIELING, C. Assessing, mapping, and quantifying cultural ecosystem services at community level. Land Use Policy, 33, 2013, p. 118-129.

PROFICE, C. C. et al. Janelas para a percepção infantil de ambientes naturais. Psicologia em Estudo, v. 18, n. 3, p. 529-539, jul./set. 2013. Disponível em: http://www.scielo.br/pdf/pe/v18n3/ v18n3a13.pdf. Acesso em: 3 set. 2020.

QUINTAS-SORIANO, C. et al. Social-ecological systems influence ecosystem service perception: a Programme on Ecosystem Change and Society (PECS) analysis. Ecology and Society, v.23, n.3, 2018. Disponível em: https://pdfs.semanticscholar.org/b04d/ f9ba1317611 ce34784886358b1bf3656626e.pdf?.ga=2.133702798.1606018776.16025370961408031317.1602537096. Acesso em: 12 set. 2020.

ROVANI, I. L et al. Socioeconomic Changes and Land Use and Land Cover of the Northern Region of Rio Grande do Sul, Brazil. Floresta Ambient., v. 27, n. 3, e20180258, 2020. Disponível em: http://www.scielo.br/scielo.php?script=sci_arttext\&pid=S2179-80872020000300103\&lng=en\&nrm= iso. Acesso: 12 out. 2020.

SANTOS, P. O. S. dos; PAULO, M. A.L. Invertendo a ordem geracional: a relação das crianças da zona rural de Orobó (PE) com as novas TIC's. Desidades, n. 21, p. 70-82,. 2018 . Disponível em: http://pepsic.bvsalud.org/scielo.php?script=sci_arttext\&pid=S2318-92822018000400006\&lng=pt\&n rm=iso. Acesso em: 4 nov. 2020.

SECRETARIADO DA CONVENÇÃO SOBRE DIVERSIDADE BIOLÓGICA. Panorama da Biodiversidade Global 4. Brasília: Ministério do Meio Ambiente, Secretaria de Biodiversidade e Florestas (MMA), 2014. Disponível em: www.cbd.int/GBO4 . Acesso em: 12 ago. 2020.

SINARE, H.; GORDON, L. J.; KAUTSKY ; ENFORS, E. Assessment of ecosystem services 
and benefits in village landscapes-A case study from Burkina Faso. Ecosystem services, v. 21, p.141-152, 2016. Disponível em: https://www.sciencedirect.com/science/article/pii/ S2212041616302170?via\%3Dihub. Acesso em: 3 ago. 2019.

SCHWARZ, M. L.; SEVEGNANI, L., PIERRE A. Representações da Mata Atlântica e de sua biodiversidade por meio dos desenhos infantis. Ciência e Educação, v.13, n. 3, p. 368-388, 2007. Disponível em: http://www.scielo.br/pdf/ciedu/v13n3/a07v13n3.pdf. Acesso em: 3 set. 2010.

SOUZA, L.; SILVA, E. da. Percepção ambiental do bioma caatinga no contexto escolar. Revista Iberoamericana de Educación, v. 73, n. 1, p. 67-86, 2017. Disponível em: https://rieoei.org/ historico/documentos/7694.pdf. Acesso em: 10 maio 2020.

UN. Forests pivotal to new Post-2015. Development Agenda Forests. Website of United Nations. Disponível em: https://www.un.org/esa/forests/news/2015/05/forests-pivotal-to-new-post-2015development-agenda/index.html. Acesso em: 16 maio 2019.

UN. Transforming our world: the 2030 Agenda for Sustainable Development. Objetivo 15, 2015.

UN. International Year of Forests 2011. Disponível em: https://static.un.org/esa/forests/wp-content/ uploads/bsk-pdf-manager/82_FACT_SHEET_IYF.PDF. Acesso em: 9 ago 2019.

UN. Department of Economic and Social Affairs Forests. United Nations strategic plan for forests, 2017-2030. Montreal. Quebec. Canada, 2017. Disponível em: https://www.un.org/esa/ forests/wp-content/uploads/2016/12/UNSPF_AdvUnedited.pdf. Acesso em: 18 out. 2019.

UNFCCC. The Marrakesh Accords \& The Marrakesh Declaration. Disponível em: http://unfecc. int/cop7/documents/accords_draft.pdf. Acesso em: 10 ago. 2019.

UNEP. Strategic Plan for Biodiversity 2011-2020 and the Aichi Targets. Secretariat of the Convention on Biological Diversity. Montreal. Quebec. Canada, 2010. Disponível em: https://www. cbd.int/doc/strategic-plan/2011-2020/Aichi-Targets-EN.pdf. Acesso em: 10 ago. 2019.

VIEIRA, M. R. M.; VARGAS, I. A. de; ZANON, A.M. Percepção ambiental e representações do pantanal: uma análise com alunos do $5^{\circ}$ ano do ensino fundamental, Rio Verde de Mato Grosso (MS). In: Anais do VIII EPEA - Encontro Pesquisa em Educação Ambiental. Anais [...]. Rio de Janeiro: UNIRIO; UFRRJ; UFRJ, 2015. Disponível em: http://epea.tmp.br/epea2015_anais/pdfs/ plenary/45.pdf. Acesso em: 10 mar. 2019.

ZANINI, A M. et al. Percepções de Estudantes do Sul do Brasil sobre a Biodiversidade da Mata Atlântica. Interciência, v. 45, n. 1, p. 15-22, 2020. Disponível em: https://www.interciencia.net/wpcontent/uploads/2020/02/15_6565_Com_Zakrzevski_v45n1.pdf. Acesso em: 7 ago. 2020.

ZAKRZEVSKI, S.B.; PARIS, A.V.; DECIAN, V. S. O olhar de jovens do Ensino Médio sobre o bioma Pampa. REMEA-Revista Eletrônica do Mestrado em Educação Ambiental, v. 37, n. 1, p. 68-88, 2020. Disponível em: https://periodicos.furg.br/remea/article/view/9317. Acesso em: 9 set. 2020.

ZAKRZEVSKI, S. B. B., VARGAS, C. V.; DECIAN, V. S. Perceptions of Farmers in Northern Rio Grande do Sul on Ecosystem Services Provided by Forests. Research, Society and Development, v.9, n.5. Disponível em: https://rsdjournal.org/index.php/rsd/article/view/2944. Acesso em: 12 out. 2020. 
